\title{
BMJ Open Selective prevention of cardiovascular disease using integrated lifestyle intervention in primary care: protocol of the Healthy Heart stepped-wedge trial
}

\author{
Tobias N Bonten (D) , ${ }^{1}$ Sanne Marije Verkleij (D) , ${ }^{1}$ Rianne MJJ van der Kleij, ${ }^{1}$ \\ Karin Busch, ${ }^{2}$ Wilbert B van den Hout, ${ }^{3}$ Niels $\mathrm{H}$ Chavannes, ${ }^{1}$ Mattijs E Numans ${ }^{1}$
}

To cite: Bonten TN, Verkleij SM, van der Kleij RMJJ, et al. Selective prevention of cardiovascular disease using integrated lifestyle intervention in primary care: protocol of the Healthy Heart steppedwedge trial. BMJ Open 2021;11:e043829. doi:10.1136/ bmjopen-2020-043829

- Prepublication history and additional supplemental material for this paper are available online. To view these files, please visit the journal online. To view these files, please visit the journal online (http://dx.doi org/10.1136/bmjopen-2020043829).

Received 17 August 2020 Accepted 23 June 2021

Check for updates

(c) Author(s) (or their employer(s)) 2021. Re-use permitted under CC BY-NC. No commercial re-use. See rights and permissions. Published by BMJ.

${ }^{1}$ Department of Public Health and Primary Care, Leids Universitair Medisch Centrum, Leiden, The Netherlands ${ }^{2}$ Hadoks Chronische zorg BV, Den Haag, The Netherlands ${ }^{3}$ Department of Biomedical Data Sciences, Leiden University Medical Center, Leiden, The Netherlands

Correspondence to Dr Tobias N Bonten; t.n.bonten@lumc.nl

\section{ABSTRACT}

Introduction Lifestyle interventions are shown to be effective in improving cardiovascular disease (CVD) risk factors. It has been suggested that general practitioners can play an essential role in CVD prevention. However, studies into lifestyle interventions for primary care patients at high cardiovascular risk are scarce and structural implementation of lifestyle interventions can be challenging. Therefore, this study aims to (1) evaluate (cost-)effectiveness of implementation of an integrated group-based lifestyle programme in primary care practices; (2) identify effective intervention elements and (3) identify implementation determinants of an integrated group-based lifestyle intervention for patients with high cardiovascular risk.

Methods and analysis The Healthy Heart study is a non-randomised cluster stepped-wedge trial. Primary care practices will first offer standard care during a control period of 2-6 months, after which practices will switch (step) to the intervention, offering participants a choice between a group-based lifestyle programme or standard care. Participants enrolled during the control period (standard care) will be compared with participants enrolled during the intervention period (combined standard care and group-based lifestyle intervention). We aim to include 1600 primary care patients with high cardiovascular risk from 55 primary care practices in the area of The Hague, the Netherlands. A mixed-methods process evaluation will be used to simultaneously assess effectiveness and implementation outcomes. The primary outcome measure will be achievement of individual lifestyle goals after 6 months. Secondary outcomes include lifestyle change of five lifestyle components (smoking, alcohol consumption, diet, weight and physical activity) and improvement of quality of life and self-efficacy. Outcomes are assessed using validated questionnaires at baseline and 3, 6, 12 and 24 months of follow-up. Routine care data will be used to compare blood pressure and cholesterol levels. Costeffectiveness of the lifestyle intervention will be evaluated. Implementation outcomes will be assessed using the REAlM model, to assesses five dimensions of implementation at different levels of organisation: reach, efficacy, adoption, implementation and maintenance. Determinants of adoption and implementation will be assessed using focus groups consisting of professionals and patients.
Strengths and limitations of this study

- This study uses a stepped-wedge non-randomised trial design. By doing so, all participating practices can sequentially benefit from the effects of offering a lifestyle intervention.

- Effectiveness and implementation outcomes are assessed using a mixed-methods process evaluation to simultaneously assess effectiveness and implementation outcomes in a real-life setting.

- Effectiveness will be evaluated on the practice level, providing practical information about which and how many patients with high CVD risk participate in a group-based lifestyle intervention when offered.

- Focus groups with both healthcare professionals and patients will provide insights in the 'black-box' detailing how to motivate patients with high CVD risk to achieve a healthier lifestyle.

- The lack of randomisation is a limitation given the groups may differ at baseline. Analyses will therefore be corrected for relevant baseline variables.

Ethics and dissemination This study is approved by the Ethics Committee of the Leiden University Medical Center (P17.079). Results will be shared with the primary care group, healthcare providers and patients, and will be disseminated through journal publications and conference presentations.

Trial registration number NL60795.058.17. Status: preresults

\section{BACKGROUND}

Cardiovascular disease (CVD) causes substantial mortality and morbidity worldwide, making prevention of high importance. ${ }^{12}$ Of the CVD burden, $70 \%$ can be attributed to modifiable risk factors. ${ }^{3}$ For example, overweight, suboptimal diet, low physical activity and tobacco use are major contributors to CVD mortality and morbidity. ${ }^{3}$ A recent large cohort study showed that individuals with an unhealthy lifestyle (current smoking, overweight and high blood pressure) die on 
average 6 years earlier than their counterparts. ${ }^{4}$ Consequently, significant health improvements could in theory be obtained by improving these modifiable CVD risk factors.

There is clear evidence demonstrating the beneficial effects of improving lifestyle factors on CVD risk. Multiple reviews showed that lifestyle interventions are effective in reducing CVD in a high-risk population. ${ }^{5-7}$ Recently, the Framingham Offspring Study found similar results: participants with intermediate or ideal cardiovascular health were $33 \%$ less likely to develop hypertension and $25 \%$ less likely to develop CVD than individuals who had poor cardiovascular health in the past 5 years. ${ }^{8}$ Even in patients with high genetic CVD risk, a healthy lifestyle was associated with a nearly $50 \%$ lower relative risk of CVD. ${ }^{9}$ When adequately implemented, lifestyle interventions can achieve reductions in blood pressure and cholesterol levels nearing those achieved via pharmacological treatment. ${ }^{10}$ Structural implementations of cost-effective lifestyle programmes are known to be challenging. Lifestyle interventions may be cost-effective when directed at a high-risk population, so-called 'selective prevention'. An important aspect of selective prevention is finding the right patients and monitoring large patient groups. Given their frequent contact with patients, general practitioners (GPs) can play an essential role in the follow-up of high-risk patients. ${ }^{2}$ However, due to limited consultation time, motivating patients to improve their lifestyle can be challenging in everyday practice. ${ }^{11}$ Therefore, an integrated lifestyle intervention programme by a lifestyle coach could be a valuable extension to CVD prevention in primary care, especially when such a programme can be personalised and directed to the specified subgroups which may benefit most.

However, studies on (cost-) effectiveness of such integrated lifestyle intervention programmes for patients with high CVD risk in primary care are scarce. Previous lifestyle intervention studies were conducted in a controlled clinical trial setting, making it difficult to assess the impact of lifestyle programme implementations in real-life settings. Also, most of the studies on lifestyle interventions were carried out in hospital or community settings, making them less generalisable to daily primary care practice. ${ }^{12}$ Furthermore, it is unclear which factors influence and optimise implementation of lifestyle interventions for patients with high cardiovascular risk in primary care.

Therefore, the primary goals of the Healthy Heart study are to (1) evaluate (cost-) effectiveness of implementation of an integrated group-based lifestyle programme in primary care practices; (2) identify effective intervention elements and (3) identify implementation determinants of an integrated group-based lifestyle intervention for patients with high cardiovascular risk. In this article, we describe the rationale and study design of the Healthy Heart study.

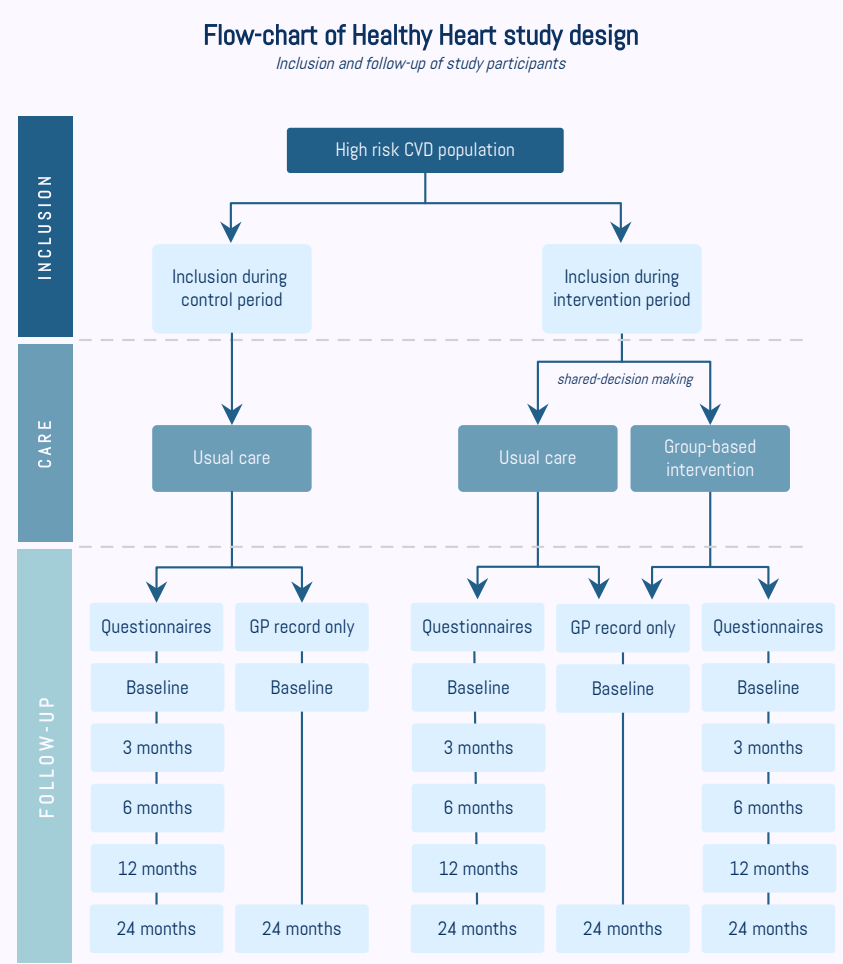

Figure 1 Flowchart of the healthy Heart study design: inclusion and follow-up of study participants in each primary care practice (cluster). CVD, cardiovascular disease; GP, general practitioner.

\section{METHODS}

\section{Study design}

This is an non-randomised cluster stepped-wedge trial intended to assess the effectiveness of a group-based lifestyle programme on practice level. We designed this study as a clustered trial, meaning the intervention is implemented at the general practice level, rather than the individual level. During the active study recruitment period (1 year), participating practices (clusters) implement the lifestyle programme in 'steps' of 2-6 months. Following the stepped-wedge approach, the 2-6-month period prior to implementation serves as the control period. During this control period, patients are offered standard preventive care only, whereas patients included during the intervention period are offered the groupbased lifestyle programme. Figure 1 shows a flowchart of the study design. Based on shared-decision making with their practice nurse or GP, patients proceed either with only standard preventive care only or with standard and group-based interventions. To assess effectiveness on the practice (cluster) level, two observation periods will be compared: the control period (all patients receiving standard preventive care) and the intervention period (all patients receiving combined standard preventive care and the group-based intervention).

\section{Setting}

This study is part of a collaboration between the Department of Public Health and Primary Care of the Leiden 
University Medical Center and Primary Care Group The Hague (Hadoks), situated in Leiden and The Hague, the Netherlands. Hadoks is a primary care collaboration of $500 \mathrm{GPs}$, taking care of 720000 patients in the area of The Hague. Daily management of Hadoks is performed by management staff members and specialised practice nurses. To make participating for primary caregivers compatible with daily practice, the start date of the study will be agreed on beforehand following discussion with the GPs, practice nurses and decision-makers of the primary care group. The lifestyle programme will be implemented in the participating primary care practices as part of routine care. As a result, recruitment of patients will be initiated in primary care practices connected to the care group and not through recruitment by the research team.

\section{Study population}

Inclusion criteria for primary care practices are:

- Already offering the structured CVD prevention protocol, which is usual care in The Netherlands.

Inclusion criteria for patients are based on International Classification of Primary Care (ICPC) and Anatomical Therapeutic Chemical (ATC) codes:

- Hypertension uncomplicated (K86), hypertension complicated (K87) or lipid disorder (T93)

And using

- One of the following ATC codes: cardiac therapy (C01), antihypertensive drugs (C02), diuretic drugs (C03), peripheral vasodilators $(\mathrm{C} 04)$, vasoprotective drugs (C05), beta-blocking agents (C07), calcium channel blockers (C08), agents acting on the reninangiotensin system (C09) or lipid-modifying agents (C10).

- Capable of providing informed consent.

This selection corresponds to patients with a 10-year cardiovascular risk of $\geq 10 \%$ according to Dutch Cardiovascular Risk Management guidelines from 2012, the most recent version available at study start. ${ }^{13}$

In each practice, high-risk patients being treated using the structured CVD prevention protocol are invited by their practice nurse or GP to participate in this study during a primary care consultation. Selection of highrisk patients for the structured CVD prevention protocol is part of routine care, carried out by GPs, practice nurses and qualified staff members of the primary care group select, and is performed without any involvement of the researchers to ascertain unbiased selection of participants. $^{13}$

Patients with an ICPC code diabetes mellitus (T90) or pre-existing CVD are excluded from the structured CVD prevention protocol as they are already selected for the diabetes mellitus or the CVD structured primary care protocol, respectively. Also, the lifestyle intervention programme is not reimbursed for patients with diabetes without CVD at study start. Exclusion of patients with CVD is based on the following ICPC codes: ischaemic heart disease with angina pectoris (K74), acute myocardial infarction (K75), ischaemic heart disease without angina pectoris (K76), transient cerebral ischemia (K89), cerebral infarction (K90.03), intermittent claudication (K92.01) and aortic aneurysm (K99.01). Furthermore, based on the judgement of the GP, patients with significant comorbidities like dementia or cancer in the palliative phase and patients living in nursing homes are excluded from participation in the structured CVD prevention protocol. Active study recruitment started in July 2017 and continued until May 2019. Participants will be followed for 2 years, so the last follow-up data are expected in May 2021.

\section{Usual care}

During the control period, patients are offered usual preventive care only. According to the structured CVD prevention protocol, standard preventive care consists of one to four individual consultations per year with a practice nurse specialised in cardiovascular care and trained in lifestyle education. During these standard care consultations, practice nurses can set personalised goals for lifestyle change and use motivational interviewing techniques. As is the case in standard care, the consultation frequency and intensity of lifestyle coaching are left to the practice nurses. Practice nurses are all trained according to the Dutch standards for primary care nurses, which include competencies in prevention and lifestyle counselling at each visit.

\section{Group-based lifestyle intervention}

Patients included during the intervention period proceed, based on shared-decision making, with usual preventive care only or follow the integrated group-based lifestyle programme on top of usual care. An overview of the lifestyle programme is shown in figure 2 and online supplemental table S1. The group-based intervention is offered to patients by the practice nurse or GP during a standard preventive care consultation. The practice nurse or GP refers patients to the lifestyle coach using their online registration system and the lifestyle coach uses the same registration system to report progress back to the practice nurse and GP. Only lifestyle coaches who are certified and trained by the Dutch Association for Lifestyle Coaches are included in this study. Lifestyle coaches are members of the Professional Association of Lifestyle Coaches in the Netherlands. They completed a post-graduate training course in lifestyle coaching at the Dutch Academy for Lifestyle and Health. Patients who choose to follow the lifestyle programme attend eight group sessions over the course of 5 months in groups of 8-10 people (online supplemental table $\mathrm{S} 1$ ). During group sessions, all aspects of lifestyle change (smoking, alcohol intake, dietary quality, overweight, physical activity and stress management) are discussed. Each session consists of providing information on lifestyle components, motivation of participants and setting of realistic personal goals. Given the influence of psychosocial factors on CVD risk and lifestyle change, stress management is likewise discussed. ${ }^{12}$ 


\section{REFERRAL PATHWAY}

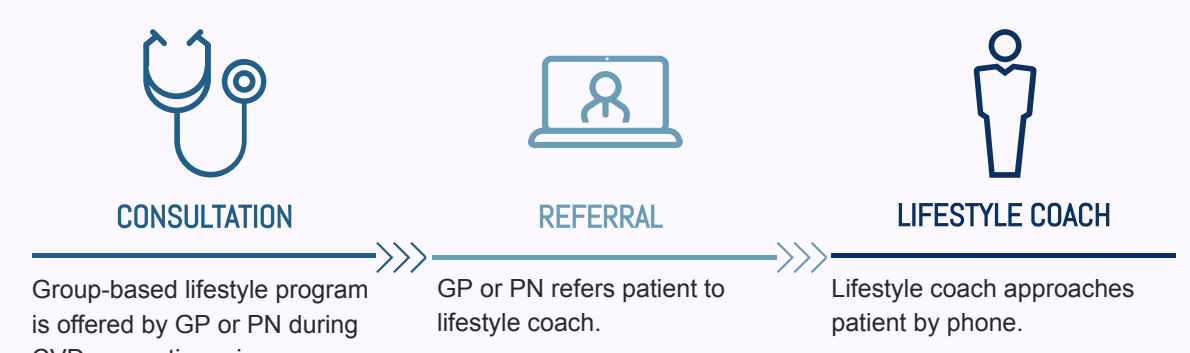

CVD preventive primary care consultation.

Figure 2 Overview of the organisation and content of the group-based lifestyle intervention. CVD, cardiovascular disease; GP, general practitioner; PN, practice nurse.

Thus, despite sessions being group-based, lifestyle goals are individualised. In addition, patients have three individual sessions with their lifestyle coach during which their personal goals are discussed and coached. Importantly, ways of maintaining lifestyle change are addressed during all sessions. The advice and activities making up the lifestyle programme are in line with the 'lifestyle' care programmes of the Dutch College of General Practitioners. $^{14}$

The primary care group implements the intervention at a neighbourhood level, making it possible for patients to follow the lifestyle programme in their neighbourhood. 
Table 1 Measurements of the Healthy Heart study.

\begin{tabular}{|c|c|c|c|c|c|}
\hline \multirow[b]{2}{*}{ Outcome } & \multirow[b]{2}{*}{ Measurement } & \multicolumn{4}{|c|}{ Frequency (months) } \\
\hline & & 0 & 6 & 12 & 24 \\
\hline $\begin{array}{l}\text { Baseline } \\
\text { characteristics }\end{array}$ & $\begin{array}{l}\text { Age, gender, body weight, length, nationality, } \\
\text { educational status, living status, working status, living } \\
\text { area }\end{array}$ & & & & \\
\hline Primary outcome & Goal setting & & & & \\
\hline \multicolumn{6}{|l|}{ Secondary outcomes } \\
\hline \multicolumn{6}{|l|}{ Lifestyle changes } \\
\hline Physical activity & SQUASH & & & & \\
\hline Diet & DHD-FFQ, DHD index & & & & \\
\hline Quality of life & SF-12, EQ-5D-5L & & & & \\
\hline General self-efficacy & General Self-Efficacy Scale & & & & \\
\hline Food security status & Six-item short form & & & & \\
\hline Routine care data & $\begin{array}{l}\text { Measurement results (blood pressure + cholesterol } \\
\text { levels) }\end{array}$ & & & & \\
\hline \multicolumn{6}{|l|}{ Cost-effectiveness } \\
\hline QALYs & EQ-5D-5L & & & & \\
\hline Healthcare use & $\begin{array}{l}\text { GP, hospital, dietician, physiotherapist, lifestyle coach, } \\
\text { homecare }\end{array}$ & & & & \\
\hline
\end{tabular}

BMI, body mass index; DHD-FFG, Dutch Healthy Diet Food Frequency Questionnaire; DHD index, Dutch Healthy Diet index; EQ-5D5L, 5-level EQ-5D; GP, general practitioner; QALYs, quality-adjusted life years; RE-AIM, Reach Effectiveness Adoption Implementation Maintenance; SF-12, short form-12; SQUASH, Short Questionnaire to Assess Health-enhancing physical activity.

Group sessions take place during daytime or in the evening, making the programme accessible for both employed and unemployed participants. The lifestyle intervention is covered by health insurance companies and free of charge for participating patients.

\section{Measurements}

All measurements and time points are depicted in table 1. Data from each participant will be extracted from primary care practices (routine care data from GP records) and are used to compare results between patient groups.

Baseline characteristics are assessed through questionnaires and include age, gender, ethnicity (country of birth and country of birth of parents), educational status (highest completed education), living status (alone, with partner or children), working status (currently employed yes or no) and current living area (neighbourhood).

At baseline, patients are asked if they have an individual lifestyle goal on one of the five lifestyle components: smoking, alcohol intake, diet, weight and physical activity. Motivation and self-confidence in lifestyle change are registered using the 'lifestyle' module of the Dutch
College of General Practitioners. ${ }^{14}$ Patients rank their motivation and self-confidence on a scale from 0 to 10 . During follow-up at 3 and 6 months, patients are asked whether they achieved their individual lifestyle goals (yes or no).

Participants are also given the option to provide informed consent for data of their GP record only, without filling in questionnaires. This option was built-in to include a population as broad as possible without losing participants reticent to fill in a questionnaire. ${ }^{14} \mathrm{At}$ baseline and again during all follow-up visits, smoking status, alcohol intake, dietary quality, weight and physical activity are measured and registered using questionnaires. Smoking status is assessed using the 7-day point prevalence of abstinence. Additionally, the 6-month prevalence of abstinence is registered to assess prolonged cessation of smoking. ${ }^{15}$ The Dutch Healthy Diet Food Frequency Questionnaire and Dutch Healthy Diet index (DHD index) are used to assess dietary quality and alcohol intake. ${ }^{1617}$ Bodyweight and waist circumference are self-reported and extracted from routine care data. 
Physical activity is assessed using the Short Questionnaire to Assess Health-enhancing physical activity in metabolic equivalents of tasks in hours per week. ${ }^{18}$

Quality of life and self-efficacy are measured at baseline and during follow-up at 6, 12 and 24 months using the short form-12 (SF-12), 5-level EQ-5D (EQ-5D-5L) and the Dutch version of the General Self-Efficacy Scale. ${ }^{19-21}$

Healthcare utilisation during the past few months (hospital, GP or other healthcare provider visits) are registered at baseline and during follow-up at 6, 12 and 24 months.

Food security status, the extent to which one has economic access to nutritious food, is measured at baseline using the Six-Item Short Form of the U.S. Household Food Security Survey Module. ${ }^{22}$ Food security status is registered as it is associated with CVD risk and can act as a barrier to a healthy diet. ${ }^{1}$

\section{Implementation}

The primary care group Hadoks implements the lifestyle intervention programme in the primary care practices. Specifically, this is done by a staff nurse specialised in CVD during a start meeting in every primary care practice. During this start meeting, the GP, practice nurse and lifestyle coach are educated on the start and content of the programme and instructed on referral logistics and registration for the study. During the programme, the staff nurse, supported by the Healthy Heart study team, is available for feedback and questions concerning the lifestyle programme or research study.

To study implementation in all practices, a mixedmethods process evaluation will be used to simultaneously assess effectiveness and implementation outcomes. We will use the RE-AIM model to examine the Reach, Effectiveness, Adoption, Implementation, and Maintenance of the intervention as described below. ${ }^{23}{ }^{24}$ Evaluation of determinants of Adoption and Implementation will be guided by the Consolidated Framework of Implementation Research. ${ }^{25}$

The reach of the intervention on practice level will be assessed using GP practice characteristics (size of patient population, located in low or high socioeconomic neighbourhood) and statistics on the number of invited practices will be provided. Patient characteristics and number of referred patients will be described to estimate the reach of the intervention on patient level.

Effectiveness of the intervention will be evaluated using the primary endpoint (achievement of individual lifestyle goals) and secondary outcomes, including quality of life, as described in the Statistical analyses section.

Adoption of the intervention will be measured by describing the proportion (participating practices and patients divided by invited practices and referred patients) and representativeness of practices and patients participating in the intervention.

Level of implementation of the intervention will be evaluated from the perspective of healthcare professionals (users; adherence) and patients (end-users; dosage received). The extent to which the intervention is implemented as intended by its developers (adherence) will be assessed through self-reported checklists at the end of each contact or group session by lifestyle coaches and 6 months after the start of the intervention period by practice nurses, GPs and lifestyle coaches. ${ }^{26}$ Received dosage of the intervention will be assessed through patient questionnaires at the end of each session.

Determinants of adoption and implementation will be assessed through focus groups with professionals and patients (both patients who choose to proceed with standard care and patients who wish to follow the group-based intervention). Focus group discussions will be recorded using a digital recorder and will be transcribed verbatim using Atlas.ti 8 Windows software. Focus group participants will be asked written and audio-recorded informed consent.

The primary care group registers the number of practices offering the intervention and the number of patients participating in the lifestyle programme. Maintenance at both the practice and patient level will be monitored through these registrations by Hadoks.

\section{Cost-effectiveness}

Cost-effectiveness of the lifestyle intervention will be evaluated. Gained quality-adjusted life years (QALYs) will be calculated comparing the EQ-5D-5L questionnaire at baseline and 6 months follow-up. ${ }^{20}$ Next to participant questionnaires about healthcare utilisation, healthcare utilisation data (number of GP and hospital visits) and associated costs will be extracted from electronic patient records in each primary care practice, quality of care registration data from the primary care group (Hadoks) and patient questionnaires, valued according to the Dutch guidelines with discounting. ${ }^{27}$

To assess cost-effectiveness, an economic evaluation will be performed from a societal perspective with 2-year time horizon. Gained QALYs will be calculated using the Dutch tariff for the EQ-5D-5L. ${ }^{20}$ A cost price analysis will be performed for the group-based intervention using data from the primary care group Hadoks, also including costs not directly related to patient care (administration, physical room for group sessions and human resources and practice and primary care group level costs). Healthcare utilisation and productivity will be estimated using the electronic patient records ${ }^{27}$ Costs will be explicitly combined using a net-benefit analysis comparing patients from the control period compared with patients from the intervention period, according to intention to treat, and corrected for the same baseline variables as in the primary analysis as described in statistical analyses section. As a secondary analyses, QALYs will be calculated using the EQ-5D visual analogue scale and the SF-12 and costs will be estimated from a healthcare perspective.

\section{Sample size}

Approximately 55 primary care practices will participate during the study period. According to quality of care 
registration data from the primary care group, these practices took care of 8428 patients with a high cardiovascular risk in May 2017. With a feasible estimated response rate of $20 \%$, and a target population of about 8000 patients, we expect to include a total of 1600 participants in our study population. ${ }^{28}$ Of these 1600 participants, we intend to include 800 participants during the control period and the same number of participants during the intervention period. Interventions leading to weight loss of $\geq 5 \%$ are known to improve cardiovascular risk factors. ${ }^{10}$ With a sample size of 1600 participants, we can detect an absolute difference of $5 \%$ between the control period and intervention period, with correction for clustering using a coefficient of variation between practices (clusters) of 0.4 , with a power of $99 \%$ at a $5 \%$ significance level. ${ }^{29}$

\section{Statistical analyses}

$\chi^{2}$ tests for categorical variables and unpaired t-tests for continuous variables will be used to compare baseline characteristics of participants who are included during the control period with participants who are included during the intervention period. The same tests will be used to compare the number of lifestyle goals set at baseline (including motivation and self-confidence) and baseline levels of the five lifestyle components, food security status, quality of life (both SF-12 and EQ-5D-5L), ability of self-management and healthcare use.

\section{Primary outcome}

To assess effectiveness on practice level of offering an integrated group-based lifestyle programme compared with offering usual care only, the primary outcome will be self-reported achievement (yes or no) of at least one individual lifestyle goal at 6 months. Achievement of lifestyle goals will be determined at 6 months as this time window covers the duration of the lifestyle programme and it is a frequently used timepoint to assess the impact of lifestyle interventions. ${ }^{12}$ We will assess the composite of all lifestyle components as the primary endpoint as we wish to avoid prioritising only one or two lifestyle components as a primary endpoint to justify the integrated nature of the intervention. Random-effects logistic regression analysis will be used to estimate the OR for achieving at least one individual lifestyle goal after 6 months for participants in the intervention period (all participants receiving combined standard care and the group-based intervention) compared with participants from the control period (all participants receiving standard care). The influence of clustering on GP practice level will be taken into account and analyses will be corrected for baseline variables age, sex, educational level, ethnicity, quality of life and baseline levels of the five lifestyle components. All participants who completed at least one of the questionnaires will be included in this analysis. For participants in the intervention period, the same analysis will be done to compare the achievement of individual lifestyle goals between participants who chose to participate in the lifestyle programme and participants who chose to continue with standard care only.

\section{Subgroup analyses}

Given CVD risk and lifestyle behaviour can vary between patients with differences in educational level, ethnicity or socioeconomic status, we will perform subgroup analyses of the primary outcome to compare participants with low versus medium-high educational level, Western versus non-Western ethnicity and from primary care practices in high versus low socioeconomic areas. ${ }^{1}$

\section{Secondary outcomes}

For the secondary outcomes, we will compare participants during the control period (standard care) with participants during the intervention period (combined standard care and group-based intervention). In addition, participants who chose to participate in the lifestyle programme will be compared with participants who decided to continue with standard care only. For these groups, the following secondary outcomes will be compared:

- At baseline: number of set goals per participant, including ranked motivation and self-confidence for those goals.

- At 3 and 6 months: number of participants that completed all their set goals.

- At 3 and 6 months: achievement of goals for the five lifestyle components separately to identify the most achieved lifestyle goal.

- At 3 and 6 months: change of lifestyle, composite of: stopping smoking, reducing alcohol intake (in glasses per week), improving dietary quality (according to the DHD index ${ }^{17}$ ), reducing overweight (weight in kilograms, waist circumference in centimetres, body mass index (BMI) in $\mathrm{kg} / \mathrm{m}^{2}$ ) and increasing physical activity (in total minutes of physical activity per week).

- At 3 and 6 months: improvement in compliance to Dutch lifestyle guidelines. A patient complies with Dutch lifestyle guidelines if they do not smoke, drink a maximum of one alcoholic beverage per day, are physically active for at least $150 \mathrm{~min}$ per week, and has a maximum score of 150 on the DHD index (compliable with complete adherence to Dutch dietary guidelines). ${ }^{14} 173031$ BMI smaller than 25 will be defined as a healthy weight.

- At 3 and 6 months: improvement of quality of life and self-efficacy. ${ }^{19-21}$

- At 12 and 24 months: sustainability of improvements achieved at 6 months regarding lifestyle change, compliance to Dutch guidelines, quality of life and self-efficacy.

For these secondary outcomes, we will use linear mixed model analysis for continuous outcomes and randomeffects binomial regression for analysis of ordinal outcomes. Analyses will model a random effects for clustering at GP practice level and for repeated participant measurements. All participants completing at least one of 
the questionnaires will be included in analysis and analyses will be corrected for the same baseline variables as in the primary outcome analysis.

Analyses will be performed using SPSS (IBM SPSS Statistics, V.25.0, IBM Corp, Armonk, NY, USA). The investigators will perform the procedures.

\section{Patient and public involvement}

We consulted Pharos, an organisation for health illiteracy, and used their feedback to improve the invitation letter to participate in this study and lifestyle intervention. The Dutch organisation for patients with CVD, 'Harteraad', was asked to serve on the board committee of the Healthy Heart study, along with policy makers of the primary care group, to make sure that study outcomes and intervention are in line with patient needs. Based on feedback of participants, paper-based online questionnaires are additionally made available. Study results will be reported in Dutch summaries to all participating patients and primary care practices.

\section{DISCUSSION}

Achieving sustainable lifestyle change in patients with high cardiovascular risk is challenging in daily practice. Previous studies on lifestyle interventions as add-ons to standard care showed them to be effective in achieving lifestyle change. ${ }^{632}{ }^{33}$ Previous studies additionally suggested GPs can play an important role in CVD prevention. ${ }^{2}$ Previous literature supporting the theory behind-and aims of our study are limited however as they have been conducted under strictly controlled research conditions, making these findings less generalisable to daily primary care practice. A strength of our study is our decision to use a stepped-wedge non-randomised trial design to assess effects in a real-life setting with simultaneous study implementation. The stepped design additional strengthens our study in that all participating primary care practices can sequentially benefit from the effects of offering a lifestyle intervention. ${ }^{34}$ A limitation of our study is that we do not randomise participants, so groups may differ at baseline. Therefore, analyses will be corrected for relevant baseline variables. Another strength is that implementation is facilitated by a primary care collaboration instead of a research team, providing a real-life setting to assess effectiveness and implementation outcomes. ${ }^{35}$

The primary effectiveness outcome will be self-reported achievement of individual lifestyle goals. Despite this appearing as a non-specific measure to assess effectiveness, previous studies show that setting individual goals improves lifestyle factors and cardiovascular risk factors such as blood pressure, cholesterol levels and BMI. ${ }^{36-38}$ In setting these goals, we did not use prespecified targets for individuals as goal setting is known to be more successful when goals are realistic in terms of the individual's capability. ${ }^{25}$ A drawback to this method is the measuring of lifestyle change using guideline targets by previous studies.
Therefore, we will also assess actual lifestyle change using validated questionnaires and guideline targets.

The limited follow-up time of 24 months is a further limitation of our study, whereas a longer follow-up could better assess long-term effects of a lifestyle change. However, the follow-up duration corresponds to many previous intervention studies in this field and most important lifestyle changes can be observed in this period. For example, the mean treatment duration of weight reduction trials in hypertensive patients was 6-36 months. ${ }^{39}$ Likewise, the median follow-up duration of trials investigating multiple risk factor interventions for primary prevention of coronary heart disease was 12 months, whereas our follow-up duration is 24 months. ${ }^{5}$ Still, a recent meta-analysis showed that lifestyle intervention in subjects at high cardiovascular risk $(\mathrm{n}=6350)$ resulted in no effect to only a modest effect on systolic blood pressure and no effect on total cholesterol after 24 months. ${ }^{40}$ This substantiates the importance of longterm follow-up in lifestyle trials benefits to ensure impact on cardiovascular outcomes. Given our goal of studying the implementation of a lifestyle intervention at practice level, we believe 24 months of follow-up to be sufficient.

We will simultaneously assess (cost-) effectiveness and implementation outcomes. This mixed-methods process evaluation combines qualitative and quantitative information, increasing the relevance of the study results. For example, quantitative data on effectiveness of the lifestyle programme can be substantiated by information from the focus-groups. This provides useful information for daily practice about how to successfully incorporate a lifestyle intervention in primary care. ${ }^{41}$ This will also support policy makers in primary care with decisions about how and to what extent to provide lifestyle interventions in primary care. In addition, insights into the 'black-box' of motivating patients with high CVD risk to achieve and maintain a healthier lifestyle will be provided by focus groups.

In conclusion, this study will provide useful information about the (cost-) effectiveness and implementation of offering a group-based lifestyle intervention for patients with high cardiovascular risk in a real-life primary care setting. These results can guide healthcare professionals and policymakers in integrating achievement of lifestyle change as a more effective part of CVD prevention in primary care. The first results are expected in 2021.

\section{ETHICS AND DISSEMINATION}

This study is approved by the Ethics Committee of the Leiden University Medical Center (P17.079). All participants provide written informed consent. Study results will be shared with key stakeholders, including the primary care group, healthcare providers and patients, and will be disseminated through journal publications and conference presentations.

Twitter Tobias N Bonten @tbonten 
Contributors SMV and TNB wrote the draft of the manuscript. SMV, TNB, KB and RMJJvdK planned and conducted the study, which meant acquisition of data and follow-up of participants. RMJJvdK contributed to the design of the implementation part of the mixed methods process evaluation. WBvdH wrote and assisted with the analyses of the cost-effectiveness evaluation plan. KB contributed to design of the study in primary care practice. MEN and NC are the supervisors of the study and critically revised the manuscript. All authors revised and approved the final manuscript.

Funding This work is supported by ZonMw grant number 531001203.

Competing interests None declared.

Patient consent for publication Not required.

Provenance and peer review Not commissioned; externally peer reviewed.

Supplemental material This content has been supplied by the author(s). It has not been vetted by BMJ Publishing Group Limited (BMJ) and may not have been peer-reviewed. Any opinions or recommendations discussed are solely those of the author(s) and are not endorsed by BMJ. BMJ disclaims all liability and responsibility arising from any reliance placed on the content. Where the content includes any translated material, BMJ does not warrant the accuracy and reliability of the translations (including but not limited to local regulations, clinical guidelines, terminology, drug names and drug dosages), and is not responsible for any error and/or omissions arising from translation and adaptation or otherwise.

Open access This is an open access article distributed in accordance with the Creative Commons Attribution Non Commercial (CC BY-NC 4.0) license, which permits others to distribute, remix, adapt, build upon this work non-commercially, and license their derivative works on different terms, provided the original work is properly cited, appropriate credit is given, any changes made indicated, and the use is non-commercial. See: http://creativecommons.org/licenses/by-nc/4.0/.

\section{ORCID iDs}

Tobias N Bonten http://orcid.org/0000-0002-7719-6182

Sanne Marije Verkleij http://orcid.org/0000-0002-0197-0688

\section{REFERENCES}

1 Arnett DK, Blumenthal RS, Albert MA, et al. 2019 ACC/AHA guideline on the primary prevention of cardiovascular disease: a report of the American College of Cardiology/American heart association Task force on clinical practice guidelines. Circulation 2019;140:e596-646.

2 Piepoli MF, Hoes AW, Agewall S, et al. 2016 European Guidelines on cardiovascular disease prevention in clinical practice: The Sixth Joint Task Force of the European Society of Cardiology and Other Societies on Cardiovascular Disease Prevention in Clinical Practice (constituted by representatives of 10 societies and by invited experts) Developed with the special contribution of the European Association for Cardiovascular Prevention \& Rehabilitation (EACPR). Eur Heart $J$ 2016;37:2315-81.

3 Yusuf S, Joseph P, Rangarajan S, et al. Modifiable risk factors, cardiovascular disease, and mortality in 155722 individuals from 21 high-income, middle-income, and low-income countries (PURE): a prospective cohort study. Lancet 2020;395:795-808.

4 Licher S, Heshmatollah A, van der Willik KD, et al. Lifetime risk and multimorbidity of non-communicable diseases and disease-free life expectancy in the general population: a population-based cohort study. PLoS Med 2019;16:e1002741.

5 Ebrahim S, Taylor F, Ward K, et al. Multiple risk factor interventions for primary prevention of coronary heart disease. Cochrane Database Syst Rev 2011;1:CD001561.

6 Álvarez-Bueno C, Cavero-Redondo I, Martínez-Andrés M, et al. Effectiveness of multifactorial interventions in primary health care settings for primary prevention of cardiovascular disease: a systematic review of systematic reviews. Prev Med 2015;76 Suppl:S68-75.

7 Hulsegge G, Looman M, Smit HA, et al. Lifestyle changes in young adulthood and middle age and risk of cardiovascular disease and all-cause mortality: the Doetinchem cohort study. J Am Heart Assoc 2016;5:e002432.

8 Corlin L, Short MI, Vasan RS, et al. Association of the duration of ideal cardiovascular health through adulthood with cardiometabolic outcomes and mortality in the Framingham offspring study. JAMA Cardiol 2020;5:549-8.

9 Khera AV, Emdin CA, Drake I, et al. Genetic risk, adherence to a healthy lifestyle, and coronary disease. N Engl J Med 2016;375:2349-58.

10 Marques-Vidal P. Comparison of lifestyle changes and pharmacological treatment on cardiovascular risk factors. Heart 2020;106:852-62.

11 Geense WW, van de Glind IM, Visscher TLS, et al. Barriers, facilitators and attitudes influencing health promotion activities in general practice: an explorative pilot study. BMC Fam Pract 2013;14:20.

12 Sisti LG, Dajko M, Campanella P, et al. The effect of multifactorial lifestyle interventions on cardiovascular risk factors: a systematic review and meta-analysis of trials conducted in the general population and high risk groups. Prev Med 2018;109:82-97.

13 Tjin-A-Ton JJS, Konings KTS. [Revision Dutch Guideline Cardiovascular Disease Prevention 2019]. Ned Tijdschr Geneeskd 2019;163:D4237.

14 Dutch College of General Practitioners. NHG-Zorgmodules Leefstijl, 2015. Available: https://www.nhg.org/sites/default/files/content/ nhg_org/uploads/nhg-zorgmodules_leefstijl.pdf [Accessed 07 May 2021].

15 Hughes JR, Keely JP, Niaura RS, et al. Measures of abstinence in clinical trials: issues and recommendations. Nicotine Tob Res 2003;5:13-26.

16 van Lee L, Feskens EJM, Meijboom S, et al. Evaluation of a screener to assess diet quality in the Netherlands. Br J Nutr 2016;115:517-26.

17 Looman M, Feskens EJ, de Rijk M, et al. Development and evaluation of the Dutch healthy diet index 2015. Public Health Nutr 2017;20:2289-99.

18 Wendel-Vos GCW, Schuit AJ, Saris WHM, et al. Reproducibility and relative validity of the short questionnaire to assess health-enhancing physical activity. J Clin Epidemiol 2003;56:1163-9.

19 Gandek B, Ware JE, Aaronson NK, et al. Cross-Validation of item selection and scoring for the SF-12 health survey in nine countries: results from the IQOLA project. International quality of life assessment. J Clin Epidemiol 1998;51:1171-8.

20 M Versteegh M, M Vermeulen K, M A A Evers S, et al. Dutch tariff for the five-level version of EQ-5D. Value Health 2016;19:343-52.

21 Bosscher RJ, Smit JH. Confirmatory factor analysis of the general self-efficacy scale. Behav Res Ther 1998;36:339-43.

22 Economic Research Service. U.S. household food security survey module: Six-Item short form, 2012.

23 Moore GF, Audrey S, Barker M, et al. Process evaluation of complex interventions: medical Research Council guidance. BMJ 2015;350:h1258

24 Glasgow RE, Vogt TM, Boles SM. Evaluating the public health impact of health promotion interventions: the RE-AIM framework. Am J Public Health 1999;89:1322-7.

25 Damschroder LJ, Aron DC, Keith RE, et al. Fostering implementation of health services research findings into practice: a consolidated framework for advancing implementation science. Implement Sci 2009;4:50.

26 Carroll C, Patterson M, Wood S, et al. A conceptual framework for implementation fidelity. Implement Sci 2007;2:40.

27 Zorginstituut Nederland. Richtlijn voor economische evaluaties in de gezondheidszorg, 2015.

28 Goldman V, Dushkin A, Wexler DJ, et al. Effective recruitment for practice-based research: lessons from the real HEALTH-Diabetes study. Contemp Clin Trials Commun 2019;15:100374.

29 Hussey MA, Hughes JP. Design and analysis of stepped wedge cluster randomized trials. Contemp Clin Trials 2007;28:182-91.

30 Gezondheidsraad. Beweegrichtlijnen 2017 [English: Physical activity guidelines, 2017.

31 Gezondheidsraad. Richtlijnen goede voeding, 2015.

32 Zhang X, Devlin HM, Smith B, et al. Effect of lifestyle interventions on cardiovascular risk factors among adults without impaired glucose tolerance or diabetes: a systematic review and meta-analysis. PLoS One 2017;12:e0176436.

33 Doughty KN, Del Pilar NX, Audette A, et al. Lifestyle medicine and the management of cardiovascular disease. Curr Cardiol Rep 2017;19:116.

34 Unni RR, Lee SF, Thabane L, et al. Variations in stepped-wedge cluster randomized trial design: insights from the patient-centered care transitions in heart failure trial. Am Heart J 2020;220:116-26.

35 Artinian NT, Fletcher GF, Mozaffarian D, et al. Interventions to promote physical activity and dietary lifestyle changes for cardiovascular risk factor reduction in adults: a scientific statement from the American heart association. Circulation 2010;122:406-41. 
36 Estabrooks PA, Nelson CC, Xu S, et al. The frequency and behavioral outcomes of goal choices in the self-management of diabetes. Diabetes Educ 2005;31:391-400.

37 West SP, Lagua C, Trief PM, et al. Goal setting using telemedicine in rural underserved older adults with diabetes: experiences from the informatics for diabetes education and telemedicine project. Telemed $J$ E Health 2010;16:405-16.

38 Redfern J, Briffa T, Ellis E, et al. Patient-Centered modular secondary prevention following acute coronary syndrome: a randomized controlled trial. J Cardiopulm Rehabil Prev 2008;28:107-15.
39 Semlitsch T, Krenn C, Jeitler K, et al. Long-Term effects of weightreducing diets in people with hypertension. Cochrane Database Syst Rev 2021;2:CD008274.

40 Bergum H, Sandven I, Klemsdal TO. Long-term effects (>24 months) of multiple lifestyle intervention on major cardiovascular risk factors among high-risk subjects: a meta-analysis. BMC Cardiovasc Disord 2021;21:181.

41 Curran GM, Bauer M, Mittman B, et al. Effectiveness-implementation hybrid designs: combining elements of clinical effectiveness and implementation research to enhance public health impact. Med Care 2012;50:217-26. 